\title{
GINA, aGAIN
}

\section{Legislation to prevent genetic discrimination in employment and insurance decisions is essential so that individuals can make use of existing genetic tests to manage their own health decisions. This legislation is also imperative to protect those who volunteer for genetic research that will benefit others affected by common diseases.}

he Genetic Information Nondiscrimination Act (GINA), legislation to prevent the use of genetic information in employment and insurance decisions, has been introduced six times in various forms to the US Congress over the last 12 years and has been unanimously passed twice by the US Senate, but not by the House of Representatives. Proponents of the Act have a fresh opportunity with the newly elected $110^{\text {th }}$ Congress. The Coalition for Genetic Fairness (http://www. geneticfairness.org/), which includes the Genetic Alliance (Nat. Genet. 36, 429-430; 2004 and Nat. Genet. 38, 391; 2006), is working hard to ensure that Congress has the information it needs - and the backbone it has hitherto lacked-to pass the legislation in the face of lobbying by insurance and employer groups. Opponents have previously argued that existing legislation suffices and that there is no evidence of widespread discriminatory practices.

The journal supports the Coalition and the legislators proposing GINA. The legislation is essential if human genetic research is to continue with full public support and deliver the anticipated health benefits that underpin much of its funding. Most crucially, the human genome belongs to the whole human race, leading to the essential "principle of genetic solidarity and altruism" so aptly described by the UK Human Genetics Commission in their 2002 report on the use of personal genetic data, entitled "Inside Information" (http:// www.hgc.gov.uk). In essence, 'I get myself tested so that my results may help another affected by my disease or disease predispositions. Consequently, I will not get myself tested if it results in the loss of my (or even your) job, mortgage or health insurance. Under these circumstances, I will not be enthusiastic about blue-sky research.' These arguments, among many others, were persuasive in extending the existing UK moratorium, created in agreement with the Association of British Insurers, that prohibits the use of predictive genetic tests in insurance until at least November $1^{\text {st }}, 2011$.

For the application of existing knowledge, the potential effects of genetic inequity are chilling. According to GeneTests (http://www.genetests.org/), there are currently 1,054 clinical tests and 297 research tests for 1,351 diseases. The utility of these established tests for mutations and variants conferring certain or sizeable risks of disease depends on the confidence of those taking them that the information gained can be used to manage their health decisions without adversely affecting their employment and their access to insurance, including, of course, health coverage. Just as it is for rare disorders, genetic equity is essential in the generation of new genetic results and their application to common and complex diseases.
Arguments leveled against immediate release of results of genetic association studies claim that the significance of the results can be appreciated only after peer-reviewed expert analysis. Studies on genetic predisposition in groups cannot be applied immediately to individuals without much more investigation of the environmental and genetic context. Should the average person interpret a meta-analysis finding that the typical risk variant carries a mean odds ratio of 1.25 (for example, in the case of a recent report on Alzheimer disease (Nat. Genet. 39, 17-23; 2007)) and conclude that a variant contributing just $25 \%$ extra risk of common multifactorial disease is an insignificant influence on their life? No, because at the individual level, you either get the disease, or you don't. The variant will not act alone, either, but rather in the context of a genome full of other variants. Johanna Seddon, Mark Daly and colleagues (Nat. Genet. 38, 1055-1059; 2006) recently calculated that individuals in their study homozygous for five risk alleles at three loci associated with adult-onset macular degeneration have a 250 -fold greater risk of developing the disease than those with the lowest risk genotype.

In advising the Genetic Association Information Network of the Foundation for the National Institutes of Health (http://www.fnih. org/GAIN/GAIN_home.shtml) on its data access policy, the Genetic Alliance's position was that research progress and its translational benefits would accrue only if results were made public as soon as possible and to the widest readership. Concessions can be made to ensure that data generators get correct credit for their work, but the message was, 'More access - the sooner the better.' Bartha M. Knoppers and colleagues (Eur. J. Hum. Genet. 14, 1170-1178; 2006) find an emerging international consensus that researchers have an ethical duty to return genetic research results to the individual research participant, provided the results are valid, significant and beneficial. An exception must be made, however, in the case where the participant exercises his or her right not to know.

Often, though, it is the research participants themselves who are keenest to push researchers to find innovative and ultimately correct interpretations of genetic data that pertain to them. Indeed, some exceptionally motivated individuals may do much of the research themselves (Nat. Genet. 38, 391; 2006). Current GAIN provisions protect the anonymity of the medical records and genotypes of research subjects in accordance with institutional review board informed consent agreements. But when, in the future, will it be appropriate for research subjects themselves to access their own genotype records? Will we have enough genetically literate physicians and counselors to help them make sense of the results? 